\title{
A National Scale Infrastructure Database and Modelling Environment for the UK
}

\author{
Stuart Barr ${ }^{\mathrm{a}^{*}}$ \\ David Alderson ${ }^{\mathrm{a}}$ \\ Craig Robson ${ }^{\mathrm{a}}$ \\ Alex Otto ${ }^{a}$ \\ Jim Hall ${ }^{\mathrm{b}}$ \\ Scott Thacker ${ }^{\mathrm{b}}$ \\ Raghav Pant ${ }^{\text {ba }}$
}

\begin{abstract}
Reliable, consistent and detailed spatial-temporal data, information and knowledge on infrastructure assets is essential to inform the long term delivery of infrastructure systems. To leverage maxim utility integrated data management, analysis, modelling and visualization environments are required for this purpose. Within the UK Infrastructure Research Transitions Consortium (ITRC) a national scale spatial database, analysis and modelling system (NISMOD-DB) has been developed, that contains national scale data and information on infrastructure assets and networks, as well as, information and knowledge on the geo-temporal patterns of infrastructure demand, supply and capacity. In this paper we describe the main components of NISMOD-DB and how it can be used within infrastructure systems research.
\end{abstract}

\section{Key words: Infrastructure Systems, Database, Spatial Networks.}

\section{Introduction}

While worldwide several large scale research initiatives have been instigated into developing a new suite of infrastructure analysis and modelling tools (e.g., US National Research Council report on Sustainable Critical Infrastructure Systems, Dutch programmes on Next Generation Infrastructure and Knowledge for Climate and the Australian Critical Infrastructure Protection Modelling and Analysis (CIPMA) programme), many challenges remain to be addressed before the full suite of analytical tools are available that will provide the key insights required to develop cohesive long term critical infrastructure plans ${ }^{1}$. Amongst these, a key consideration is the availability of good quality comprehensive data and information on critical infrastructure systems $^{2}$.

\footnotetext{
${ }^{a}$ School of Civil Engineering and Geosciences, Newcastle University, Newcastle Upon Tyne NE1 7RU, UK.

${ }^{\mathrm{b}}$ Environmental Change Institute, University of Oxford, South Parks Road, Oxford OX1 3QY, UK.

*Corresponding author: Stuart Barr (stuart.barr@ ncl.ac.uk)

http://dx.doi.org/10.14453/isngi2013.proc.4
} 
However, such data and information have limited applicability unless matched by suitable data management, analysis, modelling and visualization tools ${ }^{2}$, that allow the myriad of vulnerabilities, demand/capacity constraints and lifespan renewal issues of individual infrastructure systems to be considered in association with the dependencies and/or interdependencies that exist between them ${ }^{2}$. To address this challenge in the UK, the Infrastructure Transitions Research Consortium (ITRC) has developed a prototype integrated national-scale infrastructure database and analysis system (NISMOD-DB) to support modelling of the long term capacity/demand requirements of infrastructure (NISMOD-LP) and infrastructure network risk (NISMOD-RV). In this paper we present the prototype architecture and structure of NISMOD-DB and demonstrate how it allows an analysis of large scale data and information on infrastructure systems.

\section{THE ITRC NISMOD-DB DATABASE}

\section{A. Overall Database Organisation}

Figure 1 shows the overall design of NISMOD-DB. In addition to the database itself, it also has a series of related extension modules for analysis and visualization. The database also links to the modelling software of NISMOD-LP (the modelling of the long term capacity and demand of critical infrastructure systems) and NISMOD-RV (the modelling of interdependent infrastructure systems risk and vulnerability). One of the major aims of ITRC is to compile complete national-scale representations of infrastructure systems. Thus, NISMOD-DB must be able to represent the spatial (geographic/location) characteristics of infrastructure systems as well as their non-spatial properties. To achieve this PostgreSQL RDBMS, along with its spatial extension PostGIS have been employed. PostGIS allows spatial data layers in the form of geometry tables to be encoded and also provides a wide range of spatial operators and functions that can be used to process, analyse and manipulate the spatial infrastructure systems layers ${ }^{3}$.

In the database, tables are organized and grouped in terms of their use within ITRC and then in terms of infrastructure system (Figure 1). In the case of the long term capacity/demand modelling of NISMOD-LP, standard relational tables representing the required inputs and results of the modelling undertaken are stored and organised by economic, population (demographic), energy, water (clean and waste), transport and solid waste sectors. Data for the infrastructure risk and vulnerability modelling (NISMOD-RV) is primarily stored as PostGIS spatial geometry tables (point, polylines, polygons) representing the location and spatial extent of individual infrastructure system features. Again, these are organised and grouped by infrastructure system; namely, spatial hazards, energy (electric transmission/distribution, gas transmission/distribution), water (supply and waste), transport (road, rail, air, sea (ports)) and solid waste (Figure 1). 


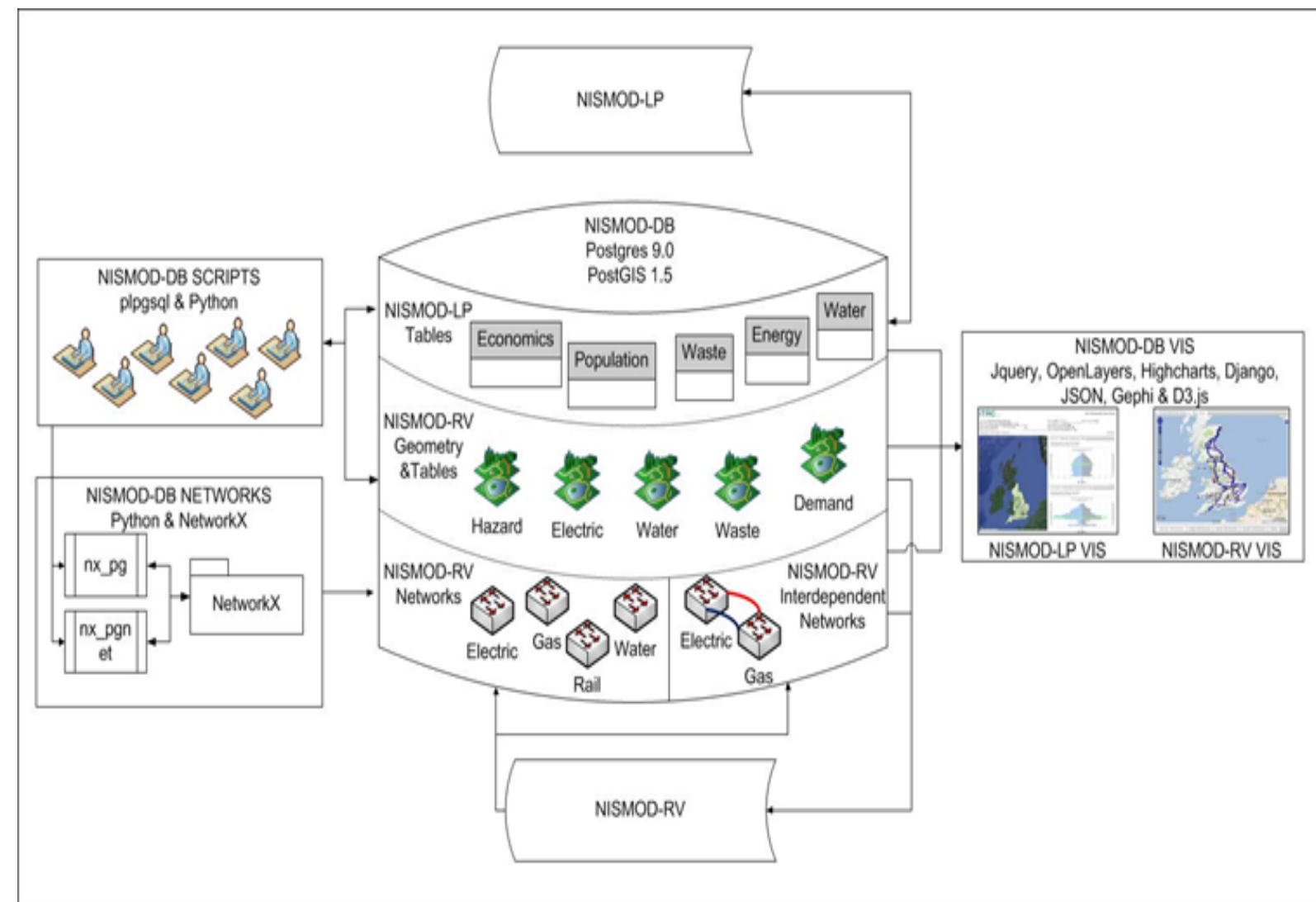

Figure 1.The overall organisation of the ITRC NISMOD-DB spatial database, analysis and visualisation framework and its linkages to other ITRC modelling software.

\section{B. Interdependent Infrastructure Network Models}

To facilitate the modelling of infrastructure networks within NISMOD-RV a bespoke interdependent network database schema has been developed in NISMOD-DB. This allows geometry tables representing infrastructure asset point and polyline features to be combined to build spatial-topological infrastructure network models (e.g., an electricity transmission network model from electricity substation points and transmission polylines), and the relationships between networks to be represented (Figure 2).

In the base schema the Graphs table keeps a record of all networks stored, while the Interdependency and Interdependency_Edges tables allow dependencies or interdependencies that exist between two networks to be stored. Any network graph is represented by a Nodes table that contains both the attributes and geometry of the node data, while Edges and Edge_Geometry tables contain the attributes and geometry of the edge data respectively. The modelling and storage of a specific network is accomplished by the specific network instance, or child tables, inheriting attributes from the parent tables of the base schema. By using inheritance, the instance tables are supplied with the minimum set of attributes, for both nodes and edges, required for a generated network model (single or interdependent) to be employed in subsequent analysis. 


\section{NISMOD-DB Scripts}

To manage and process the national data-sets held in NISMOD-DB a suite of processing scripts have been developed for database maintenance, network construction, pre-and-post network construction filtering and analysis (Figure1). Database management scripts have been written in the $S Q L$ procedural programming language plpgsql and are directly available in the database to perform administrative tasks such data import/export, creating, deleting, and setting primary and foreign key constraints between tables.

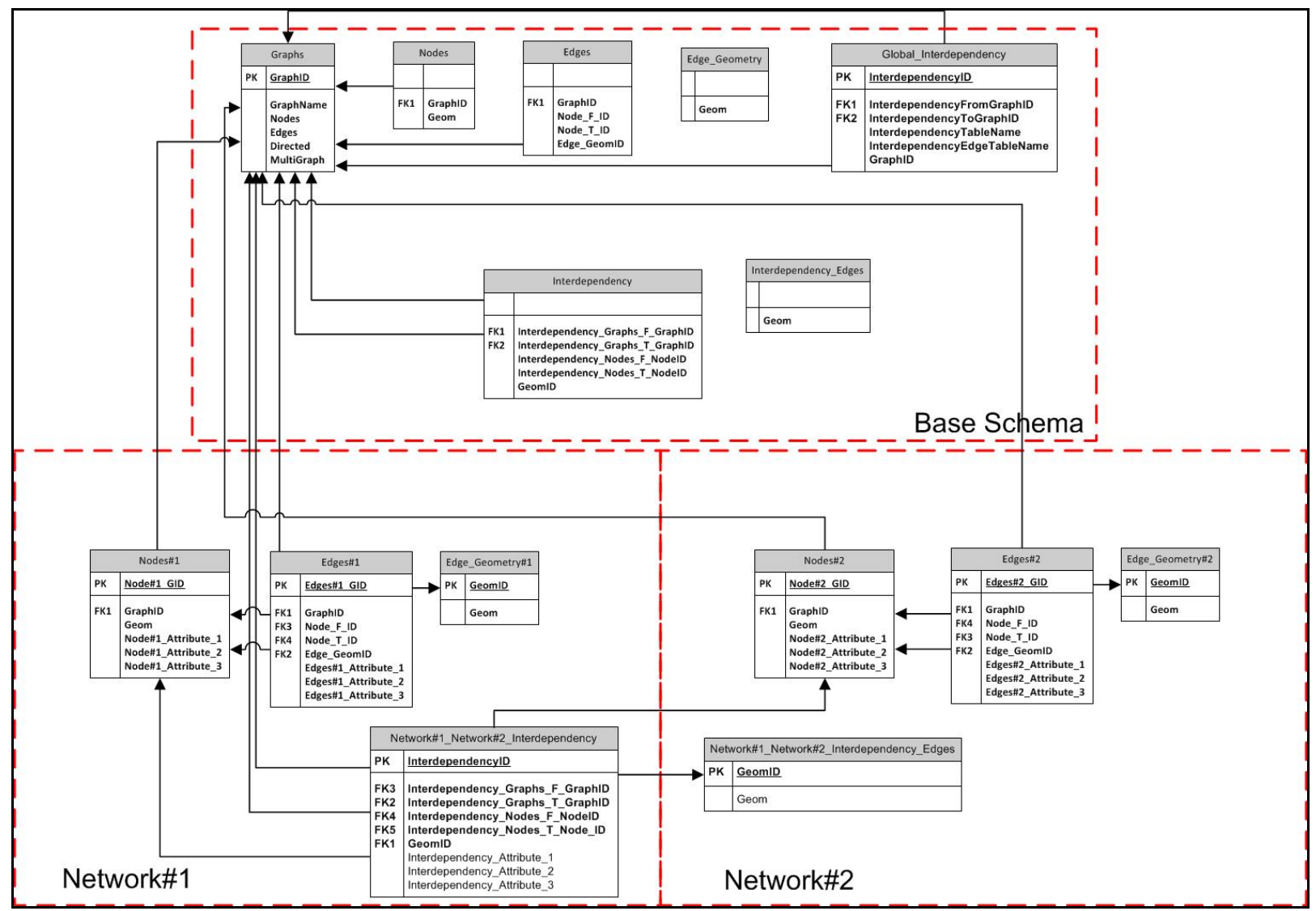

Figure 2. Entity relationship diagram showing the database schema developed for the representation of interdependent spatial infrastructure networks.

Individual or interdependent network models are created by a separate set of scripts, NISMOD-DB NETWORKS that have been developed in Python and act as a wrapper around the database (Figure 1). These scripts employ NetworkX, a Python package for the creation, manipulation, and analysis of the networks. NetworkX functions are used in two python wrapper modules; $n x \_$pgnet which converts two tables, one of nodes and one of edges stored within a PostGIS database, in to a network model that is subsequently stored using the custom network schema, and $n x \_p$ that allows node and edge tables in PostGIS to be read/written directly to/from NetworkX for analysis. 
Modules for pre-and-post network construction clean and filter data to remove or correct systematic geometry or attribute errors that result in incorrect network models being generated. Examples of such functions are EdgeToNode_SpSearch and EdgeToNode_AttLike; the former connects the end point of an unconnected edge to its nearest node within a specified search distance. The latter performs a similar task but on the basis equivalence comparison between item values of specific attributes in a node and edge table respectively. Tools such as these can be used to connect hanging edges to nodes and can be used to remedy digitisation errors. Figure 3 shows the utility of such a suite of scripts, where the original UK National Grid Gas Transmission pipes do not intersect spatially with the gas compressor. Use of the script EdgeToNode_AttLike in this instance created completed edges between the pipelines and the compressor node on the basis of a comparison of the gas pipeline and gas node names.

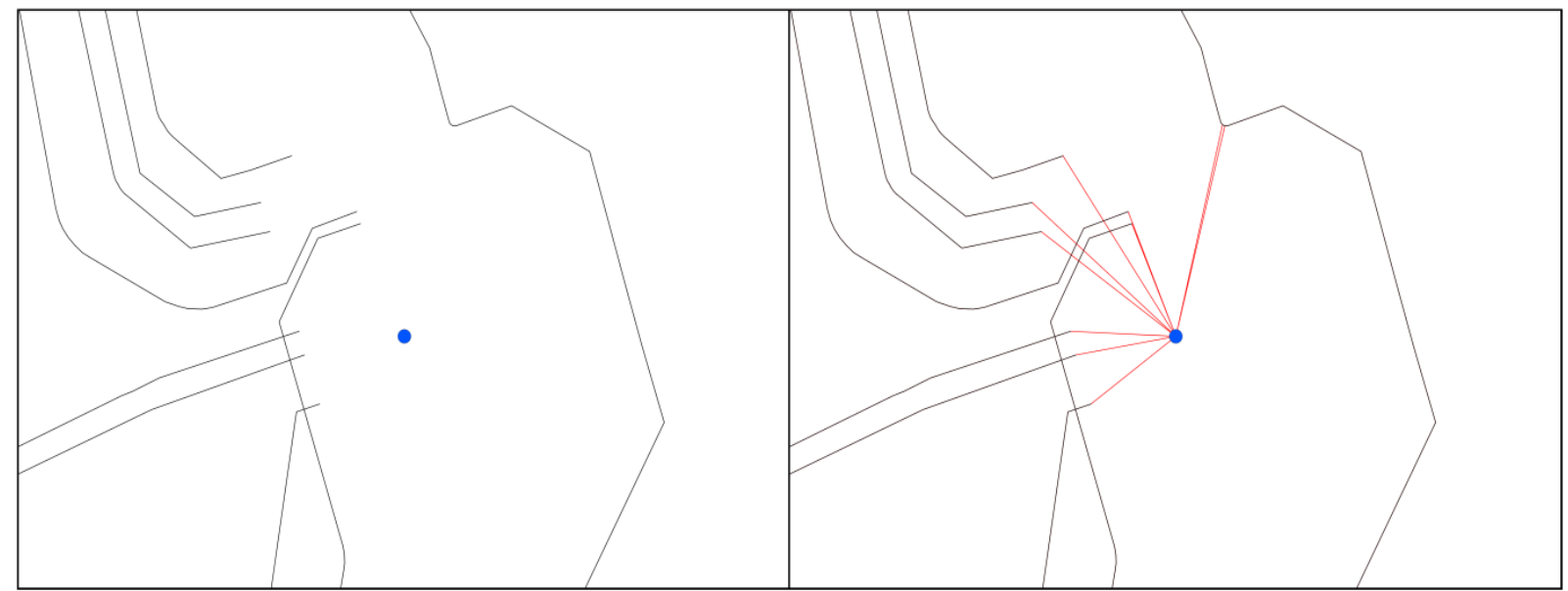

Figure 3. Use of network cleaning scripts to construct a topologically valid infrastructure network model for the national gas transmission network of the UK.

\section{NISMOD-DB Visualisation Engines}

Several prototype visualisation engines have been developed and coupled to the ITRC database; one each for NISMOD-LP and NISMOD-RV. The visualisation interfaces have been constructed using a Django-enabled web framework along with OpenLayers map client, Google Maps Application Programming Interface (API) and Highcharts. Data retrieval and querying of the database is performed via the use of synchronous AJAX requests that allow a user to directly retrieve data stored within the database and present this as a new map, plot, or time-series chart depending on the combination of the data, scenario, variable and dimension selected via the interface. Figure 4 shows two renderings of the UK National Grid Gas Transmission network; a geographic network model, and a graph rendering showing the topologically most important nodes derived by using the NetworkX Python wrappers described above. Figure 5 shows two views of the results of the NISMOD-LP modelling held in the database; in this case demographic and economic growth projections. In both cases spatial and a temporal component of the modelling are shown. 


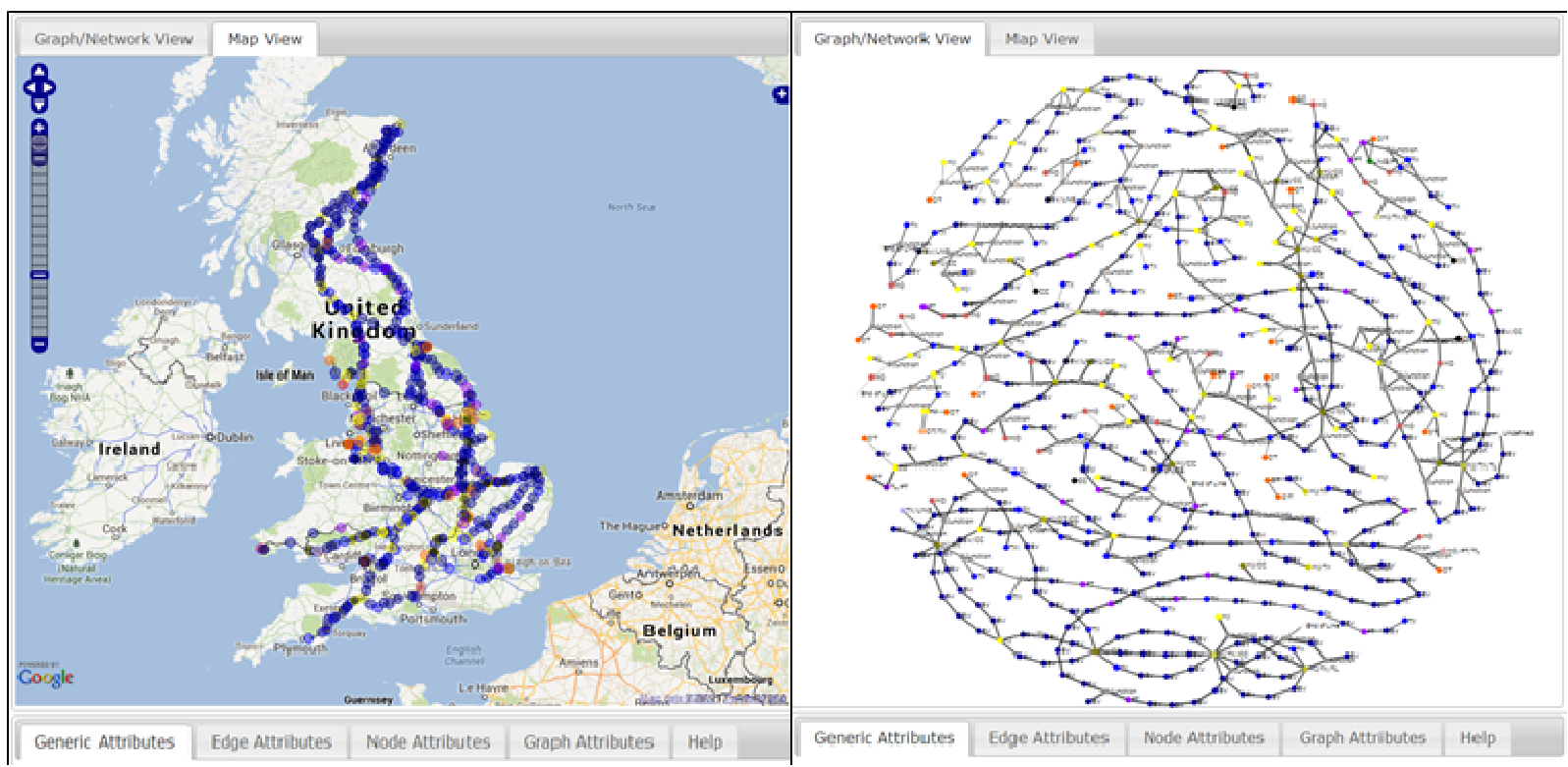

Figure 4. Two examples of the NISMOD-DB VIS outputs generated from the UK national gas troncmiccion notworl modal hold in NICMTn_DR

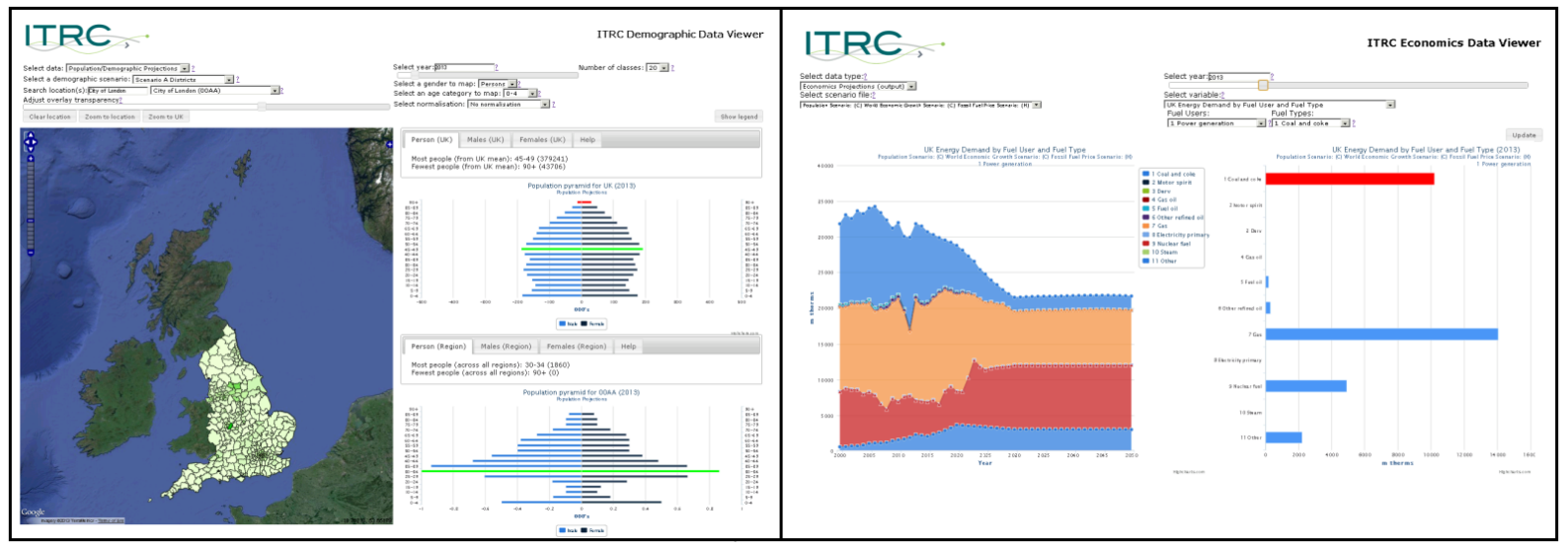

Figure 5.NISMOD-DB VIS generated views of the results derived from the capacity/demand modelling of NISMOD-LP using data stored in the NISMOD-DB database.

\section{INTEGRATED INFRASTRUCTURE ANALYSIS}

The ITRC NISMOD-DB was employed in an analysis of the dependency of the London underground tube network on electricity transmission supply using a number of failure models. NISMOD-DB PostGIS geometry tables of the London underground (stations and rail lines) and the UK National Grid electricity transmission geometry tables for the south-east of England were converted into network infrastructure models using NISMOD-DB NETWORKS. The dependency between the London underground tube network and the electricity network was generated by assuming that each tube station is powered by its geographically closest electricity substation. A basic cascading dependency failure model ${ }^{4}$ was coded for three types of electricity substation failure; random, ranked by betweenness centrality and ranked by degree ${ }^{5-7}$. In the model, a tube station fails along with its assocuited track if a substaion it is dependent on fails. Failure impact was assessed using a range of graph metrics ${ }^{8-9}$. Figure 6 shows the results for the 
three different types of failure. The map displays a single 'state' of the failure model. The plot shows that failures based on high degree and high betweenness have a more significant effect on the tube network than randomly introduced failures; implying that the London underground is more vulnerable to failure of the highly connected electricity sub-stations.

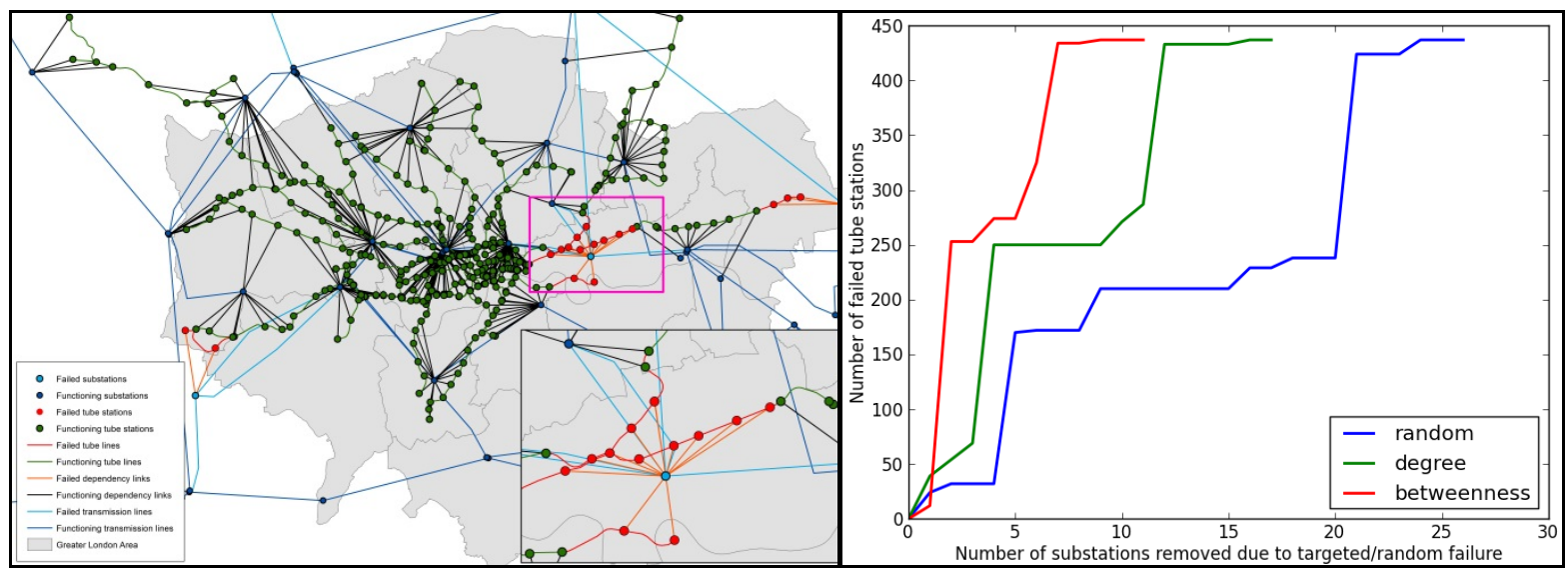

Figure 6.Interdependent infrastructure analysis using NISMOD-DB networks.

\section{CONCLUSION}

In order to manage and plan infrastructure systems at a national scale suitable analytical tools are required. This has been recognised as being particularly important in relation to an improved understanding of interdependent infrastructure systems ${ }^{2}$. As recognised by Rinaldi et $\mathrm{al}^{2}$ database systems and associated tools will play a critical role in the future management, analysis and modelling of individual and interdependent infrastructure systems. In this paper, we have described the developments that have been made in the UK ITRC project in the use of database technology for the management and analysis of national scale infrastructure systems. NISMODDB offers a flexible environment for the storage of a range of different infrastructure data, both spatial and aspatial. Furthermore, the development of a bespoke interdependent infrastructure network schema opens up the possibility of using the database as the foundation for complex infrastructure simulation modelling; results which can be readily visualised by coupling visualisation engines to the database.

\section{References}

${ }^{1}$ Council for Science and Technology, A National Infrastructure for the 21st Century. Department for Business Innovation \& Skills, UK, 2009..

${ }^{2}$ Rinaldi, S.M., Peerenboom, J.P., and Kelly, T.K., "Identifying, understanding and analysing critical infrastructure interdependencies", IEEE Control Systems, Vol. 21, No. 6, 2001, pp. 11-25. http://dx.doi.org/10.1109/37.969131

${ }^{3}$ Obe, R.O., and Hsu, L.S, PostGIS in action. Manning Publications, 2011. 
International Symposium for Next Generation Infrastructure October 1-4, 2013, Wollongong, Australia

${ }^{4}$ Johansson, J., and Hassel, H., "An approach for modelling interdependent infrastructures in the context of vulnerability analysis", Reliability Engineering \& System Safety, Vol. 95, No. 12, 2010, pp. 1335-1344. http://dx.doi.org/10.1016/j.ress.2010.06.010

${ }^{5}$ Crucitti, P., Latora, V. and Marchiori, M., "A topological analysis of the Italian power grid", Physica A, Vol. 388, No. 1-2, 2004, pp. 92-97. http://dx.doi.org/10.1016/j.physa.2004.02.029

${ }^{6}$ Dueñas-Osorio, L., Craig, J., Goodno, B., and Bostrom, A., "Interdependent Response of Networked Systems", Journal of Infrastructure Systems, Vol. 13, No. 3, 2007, pp. 185-194. http://dx.doi.org/10.1061/(ASCE)1076-0342(2007)13:3(185)

${ }^{7}$ Mishkovski, I., Biev, M., and Kocarev, L., "Vulnerability of complex networks", Communications in Nonlinear Science and Numerical Simulation, Vol. 16, No. 1, 2011, pp. 341-349. http://dx.doi.org/10.1016/j.cnsns.2010.03.018

${ }^{8}$ Albert, R., and Barabasi, A.-L., "Statistical mechanics of complex networks", Reviews of Modern Physics, Vol. 74, 2002, pp. 47-97. http://dx.doi.org/10.1103/RevModPhys.74.47

${ }^{9}$ Boccaletti, S., Latora, V., Moreno, Y., Chavez, M., and Hwang, D.U., "Complex networks: Structure and dynamics", Physics Reports, vol. 424, No. 4-5, 2006, pp. 175-308. http://dx.doi.org/10.1016/j.physrep.2005.10.009 